\title{
Références bibliographiques du dossier « Les privatisations de l'éducation »
}

\section{Bernadette Plumelle}

\section{(2) OpenEdition}

1 Journals

Édition électronique

URL : https://journals.openedition.org/ries/9227

DOI : $10.4000 /$ ries. 9227

ISSN : 2261-4265

Éditeur

France Education international

\section{Édition imprimée}

Date de publication : 1 décembre 2019

ISBN : 9782854206258

ISSN : $1254-4590$

\section{Référence électronique}

Bernadette Plumelle, «Références bibliographiques du dossier « Les privatisations de l'éducation » », Revue internationale d'éducation de Sèvres [En ligne], 82 I décembre 2019, mis en ligne le 01 décembre 2019, consulté le 14 janvier 2022. URL : http://journals.openedition.org/ries/9227 ; DOI : https:// doi.org/10.4000/ries.9227 


\section{Références bibliographiques du dossier "Les privatisations de l'éducation"}

\section{Bernadette Plumelle \\ CIEP/France Éducation international}

La privatisation de l'éducation est une question brûlante qui a fait l'objet de nombreux travaux et a suscité des publications à la fois du monde académique, des organisations internationales et de la société civile. Le sujet est clivant. Pour certaines organisations intergouvernementales telles que la Banque mondiale ou l'OCDE, l'entrée du secteur privé peut être un moyen d'améliorer la qualité de l'éducation, en favorisant la concurrence. D'autres observateurs analysent la menace que fait peser la privatisation de l'éducation en encourageant la marchandisation du savoir et en exacerbant les inégalités sociales et scolaires. La privatisation de l'enseignement s'exerce également au sein des familles avec la multiplication des cours particuliers et du tutorat (shadow education) et le développement de l'instruction à domicile.

Cette bibliographie s'attache à donner une vision panoramique de l'ensemble des phénomènes en donnant une place aux publications des organisations internationales, des chercheurs et même, à quelques reprises, du monde syndical.

Dans une première partie introductive, quelques références rappellent les évolutions des politiques éducatives et les transformations en cours des systèmes éducatifs. La section suivante propose une sélection de publications de la Banque mondiale, de l'Unesco et de l'OCDE permettant d'apprécier les différences et convergences de ces institutions sur la question de la privatisation et du développement des partenariats privé-public. Les parties suivantes ont pour sujet : les effets de la privatisation avec le libre choix des écoles, puis le soutien scolaire privé et l'instruction à domicile.

Les titres proposés dans cette sélection complètent ceux donnés par les auteurs des articles du dossier. Les résumés sont, pour l'essentiel, ceux des éditeurs et des revues.

Bibliographie arrêtée le 19 décembre 2019.

\section{ÉVOLUTION DES POLITIQUES ÉDUCATIVES}

ADAMSON Franck, ASTRAND Bjorn, DARLING-HAMMOND Linda (ed.), Global education reform: how privatisation and public investment influence education outcomes, New York: Routledge, 2016, 231 p.

L'ouvrage fournit une analyse de réformes éducatives menées dans différents contextes géographiques et situées aux deux extrémités d'un spectre idéologique. Le chapitre introductif offre 
un cadrage théorique en comparant les principes fondamentaux, les raisonnements économiques et les mécanismes éducatifs des politiques néolibérales et des politiques d'investissement public. Les études de cas examinent l'évolution historique de l'éducation au Chili, aux États-Unis, en Suède, au Canada, à Cuba et en Finlande et comparent les résultats scolaires nationaux. Un dernier chapitre résume les résultats éducatifs des différentes approches économiques et de gouvernance, ainsi que les implications politiques.

AKKARI Abdeljalil, LAUWERIER Thibaut, « The education policies of international organizations: Specific differences and convergences », Prospects, $n^{\circ} 45$, mars 2015, p. 141-157

Cet article analyse le rôle que jouent les organisations internationales dans l'orientation des réformes et des changements en matière d'éducation, sur la base d'un examen des textes que ces organisations ont produits de 1990 à 2014. L'Unesco et l'Unicef centrent leur philosophie sur une approche humaniste tandis que la Banque mondiale et l'OCDE accordent la priorité à une politique de l'éducation fondée sur l'évaluation des compétences et des résultats d'apprentissage. Au cours de la dernière décennie, cependant, il y a eu une convergence progressive de la vision de l'éducation vers les résultats d'apprentissage. Cette convergence est illustrée par l'examen de plusieurs sujets d'intérêt commun, parmi lesquels celui de la privatisation.

\section{DAVIET Barbara, "Repenser le principe d'éducation comme bien public ", Recherche et prospective en éducation, Paris : UNESCO, juillet 2016, $n^{\circ} 17,11$ p. [en ligne]}

Après la Seconde Guerre mondiale, l'éducation a été considérée comme un bien public, devant être prise en charge par l'État. Depuis, le paysage éducatif a considérablement changé et le rôle de l'État est de plus en plus contesté par les partisans du marché. Ainsi, l'approche humaniste de l'éducation perd du terrain au profit d'un discours économique envisageant l'éducation comme une marchandise. L'auteure analyse le concept de bien public et interroge sa capacité à contrer les effets de la privatisation et de la marchandisation de l'éducation. http://bit.ly/35fp64z

\section{DUTERCQ Yves (sous la direction de), Où va l'éducation entre public et privé ?, Bruxelles : De Boeck, 2011, 202 p.}

L'opposition entre public et privé a longtemps structuré la manière de voir et d'interpréter l'action dans le domaine de l'éducation. Or il existe une grande diversité des formes d'enseignement privé dans le monde. D'un pays à l'autre, les réalités sont fort différentes, à la mesure des histoires nationales en matière d'éducation, des traditions politiques et des situations économiques. Et il importe de faire la distinction entre enseignement privé et mouvement de privatisation de l'enseignement. Les contributions traitent ces questions à partir de l'étude de cas divers issus de différents espaces politiques (Angleterre, Argentine, Belgique francophone, Chili, France, Israël, Québec, Suisse).

\section{FELOUZIS Georges, MAROY Christian, VAN ZANTEN Agnès, Les marchés scolaires : sociologie d'une politique publique d'éducation, Paris : PUF, 2013, 217 p.}

Les marchés scolaires constituent un mode d'organisation de l'école dans beaucoup de pays. Les auteurs s'appuient sur les recherches internationales pour analyser les politiques de marché scolaire de plusieurs pays. Après avoir donné une définition des marchés scolaires, ils en dressent une typologie : les marchés privés, les quasi-marchés et les marchés officieux. Ils s'y intéressent ensuite en prenant en compte la diversité des contextes locaux et l'évolution des dynamiques au fil du temps. 
JONES Ken (sous la direction de), L'école en Europe : politiques néolibérales et résistances collectives, Paris : La Dispute, 2011, 226 p.

Réunissant des chercheurs européens, l'ouvrage dirigé par Ken Jones, professeur à l'Université de Londres, propose un état des lieux en Europe et analyse les transformations en cours, autonomie locale, étapes vers la privatisation, etc., et leurs effets concrets sur les dispositifs pédagogiques : accroissement des inégalités, renoncement aux savoirs. Il propose également une réflexion sur l'émergence progressive de stratégies de résistance et d'alternatives à ce nouvel ordre scolaire européen.

\section{LEWIS Laura, PATRINOS Anthony, Framework for engaging the private sector in education, Washington: World Bank, 2011, 59 p. [en ligne] \\ Ce document de la Banque mondiale présente un cadre pour impliquer le secteur privé dans l'éducation. Les auteurs considèrent que le secteur privé peut fournir aux gouvernements un moyen efficace d'accroître la qualité et l'équité de l'éducation. Après avoir analysé la façon dont les pays impliquent actuellement le secteur privé, les auteurs développent un outil pour analyser l'efficacité de cet engagement étant donné la nécessité de répondre aux éléments clés d'une offre scolaire efficace. http://bit.ly/2r4Als6}

LOCATELLI Rita, "L'éducation comme bien public et bien commun : remodeler la gouvernance de l'éducation dans un contexte en mutation ", Recherche et prospective en éducation, février 2018, $n^{\circ} 22,18$. [en ligne]

Le concept d'éducation comme bien public a longtemps été un principe essentiel du discours sur le développement de l'éducation dans le monde, en vertu duquel les institutions publiques sont premières responsables de l'offre éducative et de son financement. Mais la diversification croissante des acteurs et des sources de financement, à tous les niveaux d'enseignement, change la donne. L'auteure invite à repenser le principe d'éducation comme bien public à la lumière des tendances à la privatisation et à la commercialisation de l'éducation. http://bit.ly/2LZFWg6

\section{ZAJDA Joseph ed., Second international handbook on globalization, education and policy research, Dordrecht: Springer, 2015, 854 p.}

L'ouvrage collectif présente une vue d'ensemble des développements de l'éducation et de l'évolution des politiques au cours de la dernière décennie. Organisé en deux parties, il examine d'abord la mondialisation et les réformes des politiques en matière d'éducation. Il offre ensuite une perspective comparative qui évalue la relation ambivalente et problématique entre les réformes de la mondialisation, de l'État et de l'éducation au niveau mondial.

\section{LES POLITIQUES PRÔNÉES PAR LES ORGANISATIONS MULTILATÉRALES}

Banque mondiale, SABER: engaging the private sector, Washington: Banque mondiale, juin 2016, 4 p. [en ligne]

L'initiative de la Banque mondiale The Systems Approach for Better Education Results (SABER) a pour objectif de produire des données et des connaissances comparatives sur les politiques et les institutions éducatives. Elle évalue l'orientation politique des pays afin d'exploiter toutes les possibilités, y compris celles de l'enseignement privé. Cette plaquette présente l'initiative SABER comme un outil permettant d'évaluer la législation et l'orientation politique du pays " pour exploiter la capacité potentielle des acteurs non étatiques à mettre une éducation de qualité à la portée de tous ». http://bit.ly/36G5G92 
GENEVOIS Ilona, THÉVENIN Anne (ed.) Les défis du financement de l'enseignement de base : réexaminer les solutions impliquant le secteur privé, Paris : UNESCO, 2012, 49 p. [en ligne]

De nombreux pays ont instauré, dans les années 2000, la gratuité scolaire. Cet ouvrage reprend les quatre thèmes du forum du même nom, organisé en 2010 par l'Institut international de planification de l'éducation de l'Unesco : les dépenses des ménages et la barrière économique à l'accès à l'éducation, les politiques de gratuité pour l'enseignement primaire, l'offre privée d'éducation pour l'enseignement de base, et les partenariats public-privé. Il vise à réexaminer les solutions impliquant le secteur privé dans le financement de l'éducation de base. http://bit.ly/35qd7kw

MOUMNE Rolla, SAUDEMONT Charlotte, Overview of the role of private providers in education in light of the existing international legal framework: investments in private education: undermining or contributing to the full development of the human right to education? Paris : UNESCO, 2015, 32 p. [en ligne]

Ce document vise à donner une vue d'ensemble du rôle des prestataires privés d'éducation au regard du droit à l'éducation et du cadre juridique international. Une première partie rappelle brièvement la nature des obligations qui incombent aux États pour la mise en œuvre du droit à l'éducation. Les trois parties suivantes présentent les différents types d'enseignement privé, ainsi que les nouveaux défis liés à la privatisation et à la marchandisation de l'éducation, soulignant les impacts, positifs et négatifs du phénomène. Enfin, la dernière partie propose des solutions juridiques pour éviter les violations du droit à l'éducation. http://bit.ly/2RJntro

OCDE, Public and private schools: how management and funding relate to their socio-economic profile, Paris: OCDE, 2012, 108p. [en ligne]

Dans la plupart des pays qui participent à l'enquête PISA, le milieu socioéconomique moyen des élèves qui fréquentent des écoles privées est plus favorisé que celui des élèves des écoles publiques. Pourtant, dans certains pays, il y a peu de différences entre les profils socio-économiques des écoles publiques et privées. Une analyse des résultats de PISA montre que si la prévalence des écoles privées dans un pays n'est pas liée à la stratification socio-économique au sein du système scolaire, le niveau de financement public des écoles privées est le suivant : plus la proportion du financement public allouée aux écoles privées est élevée, plus le fossé socio-économique entre les écoles publiques et privées diminue. http://bit.ly/2M1UUC6

PSACHAROPOULOS George, PATRINOS Harry Anthony, Returns to investment in education: a decennial review of the global literature, Washington: Banque mondiale, avril 2018, 23 p. [en ligne]

Ce document porte sur le rendement de l'investissement dans l'éducation fondé sur la théorie du capital humain, à partir d'une base de données couvrant 139 pays. L'examen montre que le taux de rendement global moyen du secteur privé pour une année scolaire supplémentaire est d'environ $9 \%$ par an et qu'il est très stable sur plusieurs décennies. Les rendements privés de l'enseignement supérieur ont augmenté avec le temps, soulevant des problèmes de financement et d'équité. Les rendements sont plus élevés dans les pays à faible revenu. http://bit.ly/2DJmaRr 


\section{LA PRIVATISATION DE L'ÉDUCATION ET SES EFFETS}

ActionAid International, The impact of privatisation on the fulfilment of the right to education in 7 African countries: what do the Abidjan principles tell us? Londres : ActionAid International, juin 2019, 7 p. [en ligne]

Cette note de l'ONG internationale ActionAid International porte sur la discrimination liée à l'enseignement privé, les partenariats public-privé et l'égalité dans divers contextes (écoles, pays, ménages, communautés) dans sept pays africains (Ghana, Kenya, Malawi, Mozambique, Nigéria, Ouganda, Tanzanie). Elle considère que ces pays ne s'acquittent pas pleinement de leurs obligations en matière d'éducation publique gratuite de qualité, en partie à cause du sous-financement du secteur de l'éducation. Le secteur privé se développe, enracinant les inégalités sociales et conduisant à de fortes disparités en matière d'opportunités éducatives. http://bit.ly/2PCKgUB

ADAMSON Eric, "Les politiques éducatives aux États-Unis : entre privatisation et investissement public », Revue internationale d'éducation de Sèvres, décembre 2017, $n^{\circ} 76, p .91-101$ [en ligne]

Les décideurs politiques des États-Unis hésitent actuellement entre deux approches pour améliorer la qualité de l'école : des systèmes fondés sur le marché, qui privatisent la gestion des établissements, et des approches en termes de renforcement des capacités, qui intensifient les investissements publics. Cet article analyse les orientations, les mises en œuvre et les résultats de ces politiques à la Nouvelle-Orléans avec la privatisation de l'éducation par le biais des charter schools et dans le Massachusetts, dont la politique est basée sur l'investissement public. http://bit.ly/2rSzlY8

Banque mondiale, Sénégal : engager le secteur privé de l'éducation : rapport pays SABER 2016, Washington: Banque mondiale, juin 2016, 43 p. [en ligne]

L'initiative de l'Approche systémique pour de meilleurs résultats éducatifs (SABER) produit des données comparatives et des connaissances sur les politiques et les institutions éducatives, afin d'aider les pays à renforcer leur système éducatif. Ce rapport décrit la situation de l'éducation et de l'enseignement privé au Sénégal. Il traite ensuite de l'évolution comparative des politiques d'enseignement privé en détaillant les objectifs et aborde dans une dernière partie les options politiques pour le Sénégal. http://bit.ly/2YH3Sd5

BARRERA-OSORIO Felipe, PATRINOS Harry Antony, WODON Quentin (ed.), Emerging evidence on vouchers and faith-based providers in education: case studies from Africa, Latin America and Asia, Washington : World Bank, 2009, 186 p. [en ligne]

Dans de nombreux pays, une part substantielle des établissements d'enseignement est gérée par des entités privées. Cet ouvrage vise à fournir un ensemble d'études de cas sur les performances de différents types d'écoles dans le contexte des partenariats public-privé (PPP), en se concentrant principalement sur les résultats scolaires mais aussi sur certains aspects du fonctionnement des PPP, notamment le coût des écoles privées pour les élèves. http://bit.ly/2RNhpPO 
BONAL Xavier, VERGER Antoni, BALARIN Maria, et al., "Privatización educativa y globalización ", RASE : Revista de la asociación de sociología de la educación, mai 2016, vol. 9, $n^{\circ}$ 2, 308 p. [en ligne]

La privatisation de l'éducation est une tendance politique de portée mondiale qui s'est renforcée ces dernières années. Ce numéro aborde la privatisation de l'éducation au Pérou, à Buenos Aires et en Espagne. Il traite de sujets aussi variés que : 1) l'analyse de la relation entre privatisation et ségrégation, 2) les inégalités sociales résultant de l'émergence d'écoles à faible coût, 3) les changements dans le comportement de la demande dû au boom de l'offre privée, 4) le rôle de la nouvelle philanthropie dans l'offre de programmes éducatifs et son influence sur les décisions du gouvernement, et enfin 5) la stratégie du secteur éducatif privé pour résister à la renationalisation d'établissements privés. http://bit.ly/201NzbJ

CSEE : Comité syndical européen de l'éducation, The state of funding in education teachers working conditions social dialogue and trade union rights in western european countries, Bruxelles : CSEE : Comité syndical européen de l'éducation, février 2016, 74 p. [en ligne]

Publié par le Comité syndical européen de l'éducation (CSEE), qui représente 131 syndicats d'enseignants d'Europe, ce rapport rend compte des résultats de deux enquêtes menées en Europe de l'Ouest et en Europe centrale et orientale. Le rapport identifie un certain nombre de tendances, dont celle du financement de l'éducation et le poids du secteur privé dans le financement des services éducatifs. http://bit.ly/2S87mo0

HERRERA JELDRES Juan Francisco, REYES-JEDLICKI Leonora, RUIZ SCHNEIDER Carlos, "L'efficacité scolaire au Chili (1990-2014) : un nouveau modèle de privatisation de l'éducation ", Éducation et sociétés, 2016, $n^{\circ}$ 37, 2016/1, p. 113-127

Cet article se propose de rendre visible la signification du modèle d'efficacité scolaire comme dispositif théorique, éducatif et politique articulant les réformes éducatives des deux dernières décennies au Chili. Il analyse le processus historique qui conduit à sa progressive installation. Une relecture de la littérature existante sur le mouvement de l'efficacité scolaire et sa réception au Chili met en lumière une continuité entre les idées qui inspirent les actuelles politiques éducatives et le processus de privatisation initié pendant les années 1980.

Internationale de l'éducation - $5^{e}$ congrès mondial juillet 2007, La privatisation déguisée dans le secteur éducatif public. Rapport préliminaire établi par Stephen J. Ball et Deborah Youdell, Institute of education, University of London, Bruxelles : Internationale de l'éducation, 2007, 66 p. [en ligne]

Les deux auteurs s'attachent à dévoiler les effets de la privatisation dans le secteur public de l'éducation à deux niveaux : la privatisation dansl'enseignement public, "privatisation endogène » et la privatisation de l'enseignement public, « privatisation exogène ». http://bit.ly/36KbH4C

\section{MACPHERSON Ian, ROBERTSON Susan, WALFORD Geoffrey (ed.), Education, privatisation and social justice: case studies from Africa South Asia and South East Asia, Oxford: Symposium Books, 2014, 310 p.}

L'implication d'acteurs privés dans l'éducation n'est pas nouvelle mais des points critiques sont apparus durant cette décennie. Ce volume explore les formes émergentes du secteur privé dans l'éducation au travers d'études en Afrique (Kenya, Ghana, Nigéria) et en Asie (Bangladesh, Cambodge, Népal, Pakistan, Philippines, Vietnam). Ce phénomène de privatisation existe à tous les niveaux des systèmes éducatifs, de l'appareil administratif à l'élaboration des politiques, de l'accès aux structures d'éducation formelle jusqu'aux activités extra-scolaires, telles que le soutien scolaire privé. 
PEDRÓ Francesc, LEROUX Gabrielle, WATANABE Megumi, The privatization of education in developing countries? Paris : UNESCO, février 2016, 11 p. [en ligne]

Ce document de travail examine les principaux résultats de recherches empiriques sur les effets de la privatisation de l'éducation, en particulier dans les pays en développement où les États ne disposent pas de ressources suffisantes. Le développement de l'offre privée d'éducation est souvent considéré comme une voie prometteuse. Cependant les données sur les effets de la privatisation sont rares, et la qualité des écoles privées n'est souvent pas à la hauteur des attentes. Selon les auteurs, la meilleure voie serait d'améliorer la qualité des écoles publiques. Toutefois, le développement du secteur privé peut être considéré comme une solution provisoire, en mettant en place une réglementation efficace associée à une évolution vers de nouvelles formes de gouvernance. http://bit.ly/2sDoLdh

RIZVI Fazal, «Privatisation de l'éducation : tendances et conséquences », Recherche et prospective en éducation, Paris : UNESCO, octobre 2016, $n^{\circ} 18,13$ p. [en ligne]

La privatisation de l'éducation a les faveurs de nombreux gouvernements dans le monde, et elle a souvent été soutenue par des organisations intergouvernementales, des entreprises transnationales et des organisations non gouvernementales. Cette étude donne un aperçu des débats sur la privatisation de l'éducation ; elle présente les arguments avancés dans le passé pour et contre cette évolution, et en analyse les effets sur les inégalités sociales et la distribution inéquitable des chances en matière d'éducation. http://bit.ly/2YF90yq

\section{Les partenariats public-privé}

D'AIGLEPIERRE Rohen, L'enseignement privé en Afrique subsaharienne : enjeux, situations et perspectives de partenariats public-privé, Paris : Agence française de développement, août 2013, 158 p. [en ligne]

Cet ouvrage présente une analyse détaillée de la problématique de l'enseignement privé en Afrique subsaharienne et de son fonctionnement. Il montre à quel point il est important de mieux l'encadrer et de l'inciter à améliorer l'accès, la qualité et l'équité de l'éducation dans la région. Les contrats de partenariats public-privé comportent des bénéfices, mais aussi des risques potentiels. Pour moderniser les politiques publiques relatives à l'enseignement privé, il est essentiel d'analyser en profondeur la situation de l'État, des opérateurs de l'enseignement privé, des parents d'élèves et du secteur financier. http://bit.ly/2DiNjdl

TERMES Andreu, BONAL Xavier, VERGER Antoni, et al., Public-Private partnerships in colombian education: The equity and quality implications of "Colegios en concesión", Londres : PERI - Privatisation in Education Research Initiative, 2015, 82 p. [en ligne]

Les partenariats public-privé sont devenus très importants dans les réformes de l'éducation, en particulier dans les pays du Sud. L'un des modèles les plus connus est celui des " écoles charter ", qui consiste en la gestion des écoles publiques par des entreprises privées. Les défenseurs de ce modèle estiment qu'il favorise l'autonomie des établissements, le choix de l'école, la participation de la famille, ainsi qu'une concurrence accrue entre les établissements. En Amérique latine, la Colombie est l'un des pays qui a connu le plus intensément la mise en place des "écoles charter », en particulier, le programme nommé CEC (Colegios en concesión) mis en œuvre en 1999 à Bogota et destiné aux élèves des zones les plus vulnérables. http://bit.ly/34ANyMr 
UNESCO. IIPE Pôle de Dakar, LuxDev, OIF-IFEF : Institut de la Francophonie pour l'éducation et la formation, Faire des partenariats publics-privés en formation professionnelle un levier fort de l'émergence économique en Afrique : synthèse des travaux de l'atelier régional de partage et de renforcement mutuel sur les partenariats publics-privés dans la formation professionnelle en Afrique - Dakar, 19-21 mars 2018, Dakar UNESCO. IIPE Pôle de Dakar, 2018, 54 p. [en ligne]

Le développement économique de l'Afrique nécessite des compétences de qualité et une amélioration du niveau de qualification des jeunes. Parmi les thématiques de renforcement des dispositifs de formation professionnelle, la construction de partenariats public-privé est centrale. L'atelier régional de partage d'approches, d'expériences et d'outils a contribué à nourrir ce rapport, à travers une revue de différentes thématiques. http://bit.ly/2q0osLM

VERGER Antoni, MOSCHETTI Mauro, "Les partenariats public-privé comme approche des politiques de l'éducation : multiples significations, risques et enjeux ", Recherche et prospective en éducation, Paris : UNESCO, février 2017, $n^{\circ} 19,14 p$. [en ligne]

Les partenariats public-privé (PPP) sont de plus en plus perçus comme une approche innovante pour atteindre l'objectif de l'éducation pour tous. Néanmoins, les accords des PPP couvrent un large éventail d'options politiques, suivent des logiques diverses et les principes clés des PPP et les modalités qu'ils devraient revêtir font l'objet de débats ouverts. Les auteurs cherchent à définir les PPP à la fois comme une catégorie analytique et comme un instrument d'élaboration des politiques dans le secteur de l'éducation. Ils se penchent sur les origines, les principes fondamentaux et les diverses justifications des PPP, et examine les principaux enjeux et difficultés liés à la transposition de ces accords dans le champ des politiques de l'éducation. http://bit.ly/20VuOiL

\title{
La question du libre-choix des établissements scolaires
}

\begin{abstract}
BERTONI Marco, GIBBONS Stephen, SILVA Olmo, School choice during a period of radical school reform: evidence from the Academy programme, Bonn : Iza, novembre 2017, 61 p. [en ligne]

Donner aux écoles une plus grande liberté vis-à-vis du contrôle de l'État est souvent préconisé comme un moyen d'améliorer le niveau de l'éducation. Des pays comme les États-Unis, la Suède et plus récemment le Royaume-Uni se sont engagés dans des réformes qui donnent aux écoles financées par l'État une plus grande flexibilité et une plus grande autonomie en matière de budgets, de personnel, de programmes et d'enseignement. Les auteurs analysent le choc récent de la création et du développement des academies sur le système scolaire anglais en utilisant des données administratives issues des demandes des parents concernant ces écoles. http://bit.ly/2Rkbdxz
\end{abstract}

BONAL Xavier, BELLEI Cristián (ed.), Understanding school segregation: patterns, causes and consequences of spatial inequalities in education, London : Bloomsbury Publishing, 2018, 255 p.

Au cours des dernières décennies, la ségrégation urbaine, les mouvements migratoires et les politiques éducatives elles-mêmes ont engendré un processus croissant de ségrégation scolaire entre les groupes sociaux les plus défavorisés et les classes moyennes. L'ouvrage explore les schémas de ségrégation scolaire dans des pays d'Amérique du Nord et du Sud et en Europe et 
donne un aperçu des caractéristiques et des causes principales de la ségrégation scolaire. La troisième partie explore les effets de la privatisation de l'éducation à partir de trois études de cas, aux Pays-Bas avec le libre choix des écoles par les parents, au Pérou, en Espagne ou au Chili.

COMBAZ Gilles, "Concurrence entre établissements et choix de l'école : les personnels de direction mis à l'épreuve ? ", Éducation et sociétés, 2013, $n^{\circ} 31,2013 / 1$, p. 157-170

Parmi les transformations récentes qui ont affecté le fonctionnement des systèmes éducatifs de la plupart des pays occidentaux, le choix de l'école par les familles et la concurrence entre les établissements représentent deux dimensions importantes. En France, des mesures ont été prises en 2007 pour étendre la liberté de choix des familles. Ceci a contribué indirectement à mettre en concurrence les établissements par le biais des caractéristiques sociales des élèves qu'ils étaient susceptibles d'attirer. Les personnels de direction, confrontés à une pluralité de logiques contradictoires, éprouvent des difficultés à construire les compromis nécessaires pour garantir l'intérêt général de l'établissement.

EDWARDS D. Brent Jr., HALL Stéphanie M., " Escolas charter: gestao de professores e aquisicao de recursos na Colombia ", Cadernos de pesquisa, avril 2017, vol. 47, $n^{\circ} 164$, p. $442-468$

Les écoles charter sont envisagées comme solution dans les districts scolaires où l'on perçoit que les écoles publiques ont échoué alors même que les résultats de ces écoles indiquent que celles-ci ne produisent pas de meilleurs résultats académiques. Deux aspects de l'école charter attirent cependant moins d'attention que les autres : la gestion des professeurs et l'acquisition de ressources. L'article aborde ces questions à travers l'étude d'un programme d'écoles charter, à Bogotá, en Colombie. Dans ce programme daté de 1999, la ville a recruté des écoles privées ainsi que d'autres organisations privées pour gérer vingt-cinq écoles charter, qui ont été construites et bien équipées, dans des quartiers marginalisés. http://bit.ly/34puj8v

\section{FORSEY Martin, DAVIES Scott, WALFORD Geoffrey (ed.), The globalisation of school} choice?, Oxford studies in comparative education, 2008, 252 p.

À l'heure de la mondialisation et de la logique de marché, on observe une généralisation du choix laissé aux parents de l'établissement fréquenté par leurs enfants. Cet ouvrage collectif croise le regard de spécialistes de l'éducation, d'anthropologues et de sociologues pour analyser la situation dans une dizaine de pays des cinq continents : Argentine, Australie, Angleterre, Canada, États-Unis, Chine, Inde, Japon, Singapour, Israël et Tanzanie. Malgré une tendance globale, la réalité du terrain est très hétérogène et s'interprète toujours à travers les contextes sociaux et historiques locaux.

JACKSON Anne, Academies and free schools in England, Paris : UNESCO. IIPE : Institut international de planification de l'éducation, 2017, 14 p. [en ligne]

S'appuyant sur les recherches dont s'est inspirée l'Angleterre pour élaborer sa politique de décentralisation et d'autonomisation des établissements scolaires, l'auteure analyse trois éléments des réformes initiées en 2002. Un nombre croissant d'academies - établissements scolaires publics (financés par l'État) et autonomes - et de free schools a vu le jour. Deux tiers des élèves de l'enseignement secondaire et un cinquième des élèves de l'enseignement primaire y sont inscrits. L'auteure présente le contexte des réformes, explicite ce qui signifie en pratique l'autonomie pour les établissements scolaires, et examine l'impact des réformes. Elle souligne les liens entre l'autonomie et l'obligation de rendre des comptes. http://bit.ly/2Yi2FJc 
OCDE, School choice and school vouchers: an OECD perspective, Paris : OCDE, mai 2017, 27 p. [en ligne]

Le système des chèques éducation (school vouchers) permet aux familles de financer directement l'école dans laquelle leur enfant sera scolarisé. Il suscite de nombreux débats. Les partisans du libre choix de l'école défendent le droit des parents et les effets stimulants de la concurrence entre les établissements scolaires. Les critiques de ce système pensent que les élèves des milieux favorisés choisissent de préférence l'enseignement privé, que ce système génère une ségrégation sociale accrue et que les ressources publiques sont détournées au profit de l'enseignement privé. Ce document s'appuie sur les données de l'OCDE et sur une analyse de la situation dans des pays où ce système a été introduit (Belgique néerlandophone, Chili, Danemark, Estonie, Danemark, Pays-Bas) pour examiner ces questions. http://bit.ly/35rSXqt

OCDE, «Quels liens entre les politiques de libre choix des établissements et la mixité sociale à l'école? ", Pisa à la loupe, Paris : OCDE, mai 2019, 6 :p. [en ligne]

Dans presque tous les systèmes scolaires, les élèves se voient affectés dans l'établissement d'enseignement le plus proche de leur domicile. Toutefois, ces dernières décennies de nombreux pays ont adopté des réformes élargissant les possibilités de choix d'établissement pour les familles, en assouplissant le lien entre lieu de résidence et affectation scolaire. L'analyse des tendances en matière de mixité sociale et de pratiques d'admission scolaire sur plusieurs cycles de l'enquête PISA confirme la relation entre ces pratiques et la ségrégation entre établissements : les pays où la ségrégation sociale s'est le plus accentuée sont ceux où l'utilisation du lieu de résidence comme critère d'admission a reculé. http://bit.ly/20WNbZB

YOON Ee-Seul, LUBIENSKI Christopher, "How do marginalized families engage in school choice in inequitable urban landscapes? A critical geographic approach ", Education policy analysis archives, avril 2017, vol. 25, $n^{\circ} 42,26 p$.

La normalisation du choix de l'école dans les systèmes éducatifs est censée fournir plus d'options de scolarisation pour toutes les familles, en particulier celles qui n'ont pas les moyens de s'installer dans des zones riches avec des «bonnes " écoles. Dans quelle mesure ce choix de politique scolaire a-t-il atteint l'objectif de fournir plus de choix aux familles marginalisées ? Cet article examine les pratiques de choix de l'école pour les classes K-12 (enseignement primaire et secondaire), en mettant l'accent sur les positions spatiales des familles et leurs dispositions, à Vancouver au Canada. http://bit.ly/35yf8L0

\section{Privatisation ET INDIVIDUALISATION DE L’ÉDUCATION}

BARRÈRE Anne, DELVAUX Bernard (coord.), " La fragmentation des systèmes scolaires nationaux : dossier ", Revue internationale d'éducation de Sèvres, décembre 2017, $n^{\circ} 76,173 p$. [en ligne]

Les études rassemblées dans ce dossier qui portent aussi bien sur des pays (Pérou, États-Unis, Sénégal, France, Corée du Sud, pays scandinaves) que sur des réseaux d'écoles (Montessori, baccalauréat international, écoles confessionnelles en Europe) montrent une fragmentation des systèmes éducatifs. Chaque système scolaire national tend aujourd'hui à se fragmenter sur deux axes, l'un lié à la stratification sociale et l'autre à la différenciation des modèles et projets éducatifs. http://bit.ly/2Z6nfwz 


\section{Le soutien scolaire privé}

BRAY Mark, MAZAWI André E., SULTANA Ronald G.(éd.), Private tutoring across the Mediterranean: power dynamics and implications for learning and equity, Rotterdam : Sense publishers, Msid : EMCER, 2013, 221 p.

Le soutien scolaire privé représente une dépense considérable pour les ménages, même dans les systèmes qui prétendent avoir un enseignement public gratuit. Elle se joue à travers, à côté et même à l'intérieur de certains systèmes scolaires. Le soutien scolaire privé, qui apparaît comme une " éducation parallèle », fonctionne désormais comme un système et une industrie traversant les frontières nationales, régionales et de classe sociale. Les débats sur le soutien scolaire privé s'inscrivent donc dans le cadre des luttes plus vastes menées pour les fins de l'éducation dans des sociétés justes et équitables.

COLLAS Thomas, "Le public de soutien scolaire privé : cours particuliers et façonnement familial de la scolarité ", Revue française de sociologie, juillet 2013, $n^{\circ} 54-3$, p. 465-506 [en ligne]

L'auteur analyse la demande de cours particuliers en France en considérant ces cours en comparaison avec deux autres formes de shadow education - soutien gratuit et aide des proches - et dans leurs relations avec les autres actes et attitudes participant au façonnement familial des scolarités. L'article avance tout d'abord que les cours particuliers s'inscrivent dans un halo de pratiques participant à la construction familiale d'un avantage sur la scène scolaire et qu'ils participent, d'autre part, d'un mouvement d'externalisation de l'aide étroitement lié à la configuration du ménage. http://bit.ly/2LZTtnU

JERRIM John, Extra time: private tuition and out-of-school study new international evidence, Londres: Sutton trust, septembre 2017, 31 p. [en ligne]

Ce rapport met en relation les récents sondages qu'elle a menés auprès de jeunes âgés de 11 à 16 ans avec des informations issues de l'enquête PISA. Les sondages ont porté sur les cours particuliers (2 612 jeunes en Angleterre et au Pays de Galles, et 269 en Écosse). Près d'un élève sur trois en Angleterre et au Pays de Galles a eu des cours particuliers à un moment donné de sa vie. À Londres, la proportion est maintenant de près de la moitié des jeunes. À partir des données de l'enquête PISA, le rapport établit des comparaisons entre les pays sur l'importance de l'enseignement extrascolaire (shadow education) ainsi que sur l'inégalité dans l'accès à l'aide parentale pour les devoirs. http://bit.ly/34zKQGT

\section{L’éducation à domicile}

Commission européenne, EACEA : Agence executive : éducation, audiovisuel et culture, Eurydice : réseau d'information sur l'éducation en Europe, Home education policies in Europe: primary and lower secondary education: Eurydice report, Luxembourg: Office des publications de l'Union européenne, octobre 2018, 30 p. [en ligne]

L'enseignement obligatoire est-il synonyme de fréquentation d'un établissement scolaire ? Les parents ont-ils le droit d'instruire leurs enfants à la maison ? Ce rapport fournit un aperçu comparatif des politiques concernant l'instruction à domicile en Europe, dans les secteurs primaire et secondaire. Si la majorité des pays autorise l'instruction à domicile, une douzaine ne l'autorise que dans des circonstances exceptionnelles. La moitié des pays exigent que l'éducateur ait un niveau de qualifications minimal. http://bit.ly/2rHYav0 
FOSTER David, DANECHI Shadi, Home education in England, London: House of Commons Library, juillet 2019, 38 p. [en ligne]

En 2018, environ 53000 à 58000 enfants étaient scolarisés à domicile en Angleterre avec des chiffres en augmentation ces dernières années. Les parents ont le droit d'éduquer leurs enfants jusqu'à la fin de la scolarité obligatoire et les autorités locales n'ont ni pouvoir ni obligation officielle de contrôler l'offre d'enseignement à domicile. Cependant, ils ont le devoir de prendre des dispositions pour identifier les enfants ne recevant pas une éducation appropriée et d'intervenir si nécessaire. Des orientations actualisées sur l'éducation à domicile ont été publiées en avril 2019, à la suite d'une consultation lancée en avril 2018. http://bit.ly/2ElFmoP

\section{GUIGUE Michèle, SIRMONS Rébecca, L’instruction en famille : une liberté qui inquiète, Paris : L'Harmattan, 2015, 267 p.}

Cet ouvrage propose une exploration panoramique de l'instruction en famille, qui était prévue dès la loi de Jules Ferry de 1882 sur l'enseignement primaire obligatoire. La première partie s'attache à la situation française, du point de vue législatif et pratique. La seconde partie fait un bilan des recherches, principalement américaines. Cette pratique n'est-elle pas caractéristique des idées contemporaines plaçant l'enfant " au centre » et valorisant l'autonomie, la motivation et l'individualisation ? Elle interroge la place des parents, les modalités de transmission, les missions de l'école, la socialisation et la cohésion sociale.

MOLNAR Alex éd., Virtual schools in the U.S 2017, Boulder : National education policy center, avril 2017, $103 \mathrm{p}$. [en ligne]

Ce rapport annuel ( $5^{\mathrm{e}}$ édition) offre un aperçu des écoles virtuelles et des écoles hybrides (combinaison d'enseignement traditionnel et d'enseignement virtuel) aux États-Unis. Les élèves diffèrent des élèves des écoles publiques traditionnelles et ne réussissent pas aussi bien. Néanmoins, la croissance des inscriptions s'est poursuivie. Dans une deuxième partie, le rapport examine les recherches menées sur l'efficacité de ce type d'écoles dont les élèves ont des niveaux bien inférieurs à ceux des écoles traditionnelles. http://bit.ly/36M50Mp

\section{REDFORD Jeremy, BATTLE Danielle, BIELICK Stacey, Homeschooling in the United} States: 2012, NCES : National center for education statistics, avril 2017, 52 p. [en ligne]

Ce rapport fournit des estimations du nombre d'élèves scolarisés à la maison aux États-Unis en 2012 et compare ces estimations avec celles de 1999, 2003 et 2007. Il analyse les caractéristiques sociodémographiques de ces élèves ainsi que les raisons de ce choix par les parents et les ressources en ligne utilisées par les familles. Le pourcentage d'élèves âgés de 5 à 17 ans scolarisés à domicile a progressé, passant de $1,7 \%$ en 1999 à $3,4 \%$ en 2012, soit 1,8 million d'élèves. http://bit.ly/2YRpYcY

\section{ROTHERMEL Paula ed., International perspectives on home education: do we still need schools?, Basingstoke: Palgrave MacMillan, 2015, 333 p. \\ L'ouvrage rassemble des contributions de chercheurs de différentes disciplines sur l'éducation à domicile dans le monde. Des études sont proposées sur le Royaume-Uni, les États-Unis, l'Australie, Israël, l'Afghanistan, la Norvège, l'Allemagne, la Belgique, la Colombie, l'Espagne, l'Inde ou le Canada. Ce panorama permet d'explorer l'éducation actuelle en dehors de l'école et de la placer dans un contexte mondial, politique et critique.}




\title{
Abstracts
}

\section{The privatizations of education}

\author{
Coordination : Thierry Chevaillier, Xavier Pons
}

\author{
Introduction \\ The privatizations of education: Forms and challenges \\ Thierry Chevaillier, Xavier Pons
}

After presenting the issue that this dossier for the Revue internationale d'éducation de Sèvres is focused on and stabilizing certain definitions, this introduction distinguishes three main forms of privatization at work in the many education systems covered by the contributors to this issue (Chile, Ivory Coast, England, France, India, Sweden, United States, and Asian systems): privatization through specific public policies; privatization by the market; and privatization by increasingly taking into account aspirations and private individual interests. It then points to the major democratic challenges of this multifaceted growth in the modes of privatization of school, whether educational or political, such as the necessary regulation of areas of activity whose increasing independence can prove problematic.

\begin{abstract}
Understanding corporate influence in education: A review of policy influence strategies Clara Fontdevila and Antoni Verger

A growing body of research points to the increasing engagement of corporate actors in education policymaking processes, frequently in connection with the advancement of a privatization agenda. However, research on the strategies mobilized by corporate actors remains unsystematized and fragmentary. Building on the results of a literature review, this paper identifies four emerging strategies deployed by the corporate sector in education policy-shaping processes - namely knowledge mobilization, networking, supporting grassroots, and sponsoring pilot projects. Results suggest that the private sector engages in increasingly subtler forms of policy advocacy while simultaneously diversifying the forms of capital they mobilize to this purpose.
\end{abstract}

\section{Privatization by the market in Chilean education: Concepts, policies and consequences $\quad$ p. 47 Cristián Bellei \\ Privatization as an educational policy can refer to various initiatives and apply to different logics and objectives. This article proposes a conceptual approach in order to analyse these policies and demons- trates that privatization within the framework of education market policies is a special case. The Chilean example is then analysed as a paradigmatic example of educational privatization under a market-based logic. The article describes the policies that have been carried out in this country, their effects at the level of schools and the education system, and their consequences on families' choice of schools. The article concludes with a reflection on the extreme difficulty of reversing this process.}

\section{Privatization and marketization in US education}

Christopher Lubienski, T. Jameson Brewer, Jin Ah Kim

The traditional public-private sector distinctions across schooling options in the United States continue to manifest against a backdrop of constitutional concerns and deep philosophical questions surrounding the purpose of an education and how it is to be delivered. Overall, the marketization of education has relied on, and subsequently reinforced, a reimagining of education away from a public or common good to that of an individualistic good whereby schools compete in an education marketplace for the commodity and customers that are students. In what follows, we argue that a more nuanced analysis of the "privatization of education" reveals that it is, in fact, privatized public policymaking that has led to marketization and the privatization of the "purpose" of education. These have become major forces in education reform in the US. 
Sweden's radical shift in the early 1990s to a highly decentralized and market-oriented education system aimed at raising the quality and efficiency of education in terms of outcomes and costs. However, the results of the reforms are disappointing: on average, they show decreasing academic results over time and in international comparison, and increasing school segregation. Knowledge on the economic aspects is, however, lacking to a high extent. There have been efforts to mitigate the negative development by increased state control and evaluation, but the major features of the education system introduced in the 1990s remain largely intact. The aim of this article is to briefly describe privatization and marketization of the provision of education in Sweden from the early 1990s. A central question concerns the enabling and continuing defence of this exceptionally swift and far-reaching change.

\section{Privatization of education in the English school system} Anne West

In England, there is significant involvement of "private" entities in publicly funded schools, with clear evidence of "privatization".This article argues that the privatization of education services in the English context raises issues regarding accountability, costs, transparency and public process. In the first section, an overview of the English school system, past and present, is provided. Drawing on academic literature and documentary analysis, the sections that follow analyse three different types of privatisation: academies, outsourcing and the "Private Finance Initiative".

\section{Forms of privatization of education at work in Ivory Coast: From privatization to commodification}

\section{Zamblé Théodore Goin Bi, N'guessan Claude Koutou}

To respond to an ever-increasing demand for schooling, the Ivorian state asked the private sector to help it fulfil its mission of educating and training young people, in particular in secondary education. This article provides an overview of the private education system in Côte d'Ivoire, based on a mixed survey conducted in two regions. The results indicate that public-private partnership is essential to achieve schooling objectives. However, in modest and disadvantaged neighbourhoods, private establishments do not offer adequate conditions for quality education. Most teachers, who are poorly qualified and underpaid, have received no initial training. To minimize costs, promoters favour precarious labour contracts and reduce investments in the purchase of quality teaching and pedagogical tools.

\section{Privatization and the changing educational landscape in India Meera Samson}

This paper looks at the role of private players in the education sector in India, with special reference to the past two decades. It begins by focusing on private groups that are running their own schools. It then shifts the attention to private players who are currently providing inputs into government schools. These include primarily not-for-profit organizations set up or funded by for-profit organizations. These groups have been given the opportunity to enter into partnerships with the government to manage public schools, although this is comparatively limited at present. The discussion highlights the need to think about the impact that these current forms of privatization may have on the education of children and on society as a whole in India.

\section{Privatisation by default :The expansion and implications of private supplementary tutoring in Asia Mark Bray, Wei Zhang}

While some forms of privatization reflect active government decisions to shift balances from the public sector towards the marketplace, the rise of private supplementary tutoring has shifted balances by default. The tutoring sector has grown in response to demands from students and their families and through the entrepreneurial initiatives of companies, teachers and informal providers. From the perspective of mainstream schooling, private tutoring is not simply a supplementary activity that operates in parallel. This is because private tutoring influences and penetrates aspects of mainstream schooling. As such, the default form of privatization has far-reaching implications for mainstream schooling as well as for wider social and economic development. This paper focuses on Asia, but its overall messages have global relevance. 
Dilemmas and challenges of the educating state faced with the development of family education in France

Philippe Bongrand

The recent development of family education in France, although it concerns only very marginal numbers of children, is an exemplar for examining how public action can shape a phenomenon of privatization of education. In this case, the posture of the state and its scope are ambivalent: by recognizing this private form, the state gives it a public dimension; it gives it mediate coverage, but in a deprecatory form; it administers it, but in a minimal form, bound around the function of population control. Not wishing to encourage family education, the state has thus sketched out a form of public action, almost in spite of itself. This tension raises the pending question of whether this particular form of state-influenced education might generate a particular, and specifically defiant, form of socialization vis-à-vis the state.

Bibliographic references

p. 123

Bernadette Plumelle 